\title{
Membrane Nanotubes Increase the Robustness of Giant Vesicles
}

\author{
Tripta Bhatia, ${ }^{\dagger}$ Jaime Agudo-Canalejo, ${ }^{\ddagger}, \S$ Rumiana Dimova, ${ }^{\dagger}$ and Reinhard Lipowsky ${ }^{*}, \dagger$ \\ ${ }^{\dagger}$ Theory \& Biosystems, Max Planck Institute of Colloids and Interfaces, 14424 Potsdam, Germany \\ ${ }^{\ddagger}$ Rudolf Peierls Centre for Theoretical Physics, University of Oxford, Oxford OX1 3NP, U.K. \\ ${ }^{\S}$ Department of Chemistry, The Pennsylvania State University, University Park, Pennsylvania 16802, United States
}

\author{
Supporting Information
}

ABSTRACT: Giant unilamellar vesicles (GUVs) provide a direct connection between the nano- and the microregime. On the one hand, these vesicles represent biomimetic compartments with linear dimensions of many micrometers. On the other hand, the vesicle walls are provided by single molecular bilayers that have a thickness of a few
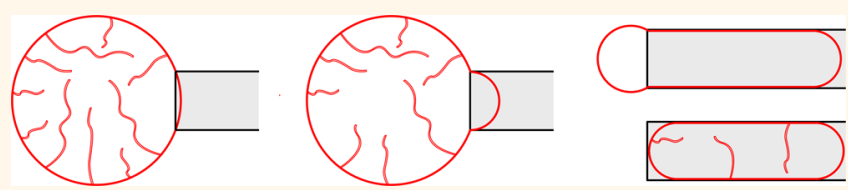
nanometers and respond sensitively to molecular interactions with small solutes, biopolymers, and nanoparticles. These nanoscopic responses are amplified by the GUVs and can then be studied on much larger scales. Therefore, GUVs are increasingly used as a versatile research tool for basic membrane science, bioengineering, and synthetic biology. Conventional GUVs have one major drawback, however: they have only a limited capability to cope with external perturbations such as osmotic inflation, adhesion, or micropipette aspiration that tend to rupture the membranes. In contrast, cell membranes tolerate the same kinds of mechanical perturbations without rupture because the latter membranes are coupled to reservoirs of membrane area. Here, we introduce GUVs with membrane nanotubes as model systems that include such area reservoirs. To demonstrate the increased robustness of these tubulated vesicles, we use micropipette aspiration and changes in the osmotic conditions applied to phospholipid membranes doped with the glycolipid GM1. A quantitative comparison between theory and experiment reveals that the response of the GUVs is governed by the membranes' spontaneous tension, a curvature-elastic material parameter that describes the bilayer asymmetry on the nanoscale. Because of their increased robustness, GUVs with nanotubes represent improved research tools for membrane science, in general, with potential applications as storage and delivery systems and as cell-like microcompartments in bioengineering, pharmacology, and synthetic biology.

KEYWORDS: biomembrane, bilayer asymmetry, giant vesicle, spontaneous tubulation, micropipette aspiration, tube retraction, spontaneous tension

iant unilamellar vesicles (GUVs) are formed by fluid bilayer membranes that separate interior aqueous microcompartments from the exterior aqueous environment. These membrane systems, which have been studied for some time, ${ }^{1}$ have attracted much recent interest, both in basic membrane science $e^{2-7}$ and in the context of artificial protocells. $^{8-10}$ The multiresponsive behavior of the GUVs includes curvature generation by polymers ${ }^{2}$ and membrane proteins, ${ }^{3,5}$ protein segregation in membrane-membrane interfaces, ${ }^{4}$ thermally driven transport of intramembrane domains, ${ }^{6}$ and membrane fission by protein crowding. ${ }^{7}$ The increased interest in GUVs is further demonstrated by the different microfluidic-based methods that have been developed during the last couple of years to produce large populations of monodisperse GUVs. ${ }^{10-16}$

In basic membrane science, one wants to stay in the regime of low membrane tension in order to mimic the behavior of cellular membranes. Likewise, when GUVs are used as microcompartments in bioengineering or synthetic biology, one would like to preserve the integrity of the membranes and to avoid membrane rupture. However, when the GUVs are osmotically inflated by hypotonic exterior solutions, ${ }^{17}$ exposed to adhesive surfaces, ${ }^{18}$ or aspirated by micropipettes, ${ }^{19}$ their membranes easily rupture in an uncontrolled manner. In contrast, the plasma membranes of eukaryotic cells, which are coupled to reservoirs of membrane area, ${ }^{20,21}$ can tolerate the same kinds of mechanical perturbations without rupture.

Here, we introduce GUVs with spontaneously formed membrane nanotubes as model systems that include such area reservoirs and demonstrate that these vesicles can resist even large mechanical perturbations without membrane rupture. We show that these GUVs tolerate several cycles of strong aspiration into and subsequent release from a micropipette as well as repeated inflation and deflation steps induced

Received: January 24, 2018

Accepted: April 9, 2018

Published: April 16, 2018 
by changes in the osmotic conditions. In addition, a quantitative analysis of the micropipette experiments reveals that the tubulated vesicles behave, to a large extent, like liquid droplets with a variable surface area and an effective interfacial tension that is provided by the spontaneous tension introduced in ref 22. The latter tension is, in fact, a curvature-elastic material parameter that depends on the bilayer asymmetry and the associated spontaneous curvature but not on the vesicle geometry.

The spontaneous tubulation of vesicles has been observed previously for a variety of lipid compositions. ${ }^{2,23}$ Here, we use binary mixtures of the phospholipid 1-palmitoyl-2-oleoyl-snglycero-3-phosphocholine (POPC) and the ganglioside GM1, together with a small amount of fluorescently labeled lipids, which provide another example for bilayer membranes that undergo spontaneous tubulation. The glycolipid GM1 has attracted much recent interest because it is abundant in all mammalian neurons ${ }^{24}$ and plays an important role in many neuronal processes and diseases. ${ }^{25}$ Furthermore, GM1 acts as a membrane anchor for various toxins, bacteria, and viruses such as the simian virus $40 .^{26}$ Our combined experimental and theoretical study implies, however, that the increased robustness of tubulated vesicles depends primarily on the bilayer asymmetry of these membranes. Therefore, any membrane with a sufficiently large asymmetry will exhibit the same robustness as the lipid membranes considered here. Recently, tubulated vesicles have also been studied by Monte Carlo simulations. $^{27}$

We aspirate the tubulated GUVs by micropipettes and study the initial aspiration process as defined in Figure 1 in a systematic manner. To the best of our knowledge, such an experimental study has not been performed previously. A quantitative comparison between theory and experiment reveals that the response of the tubulated GUVs is governed by the spontaneous tension of their membranes, a quantity that is
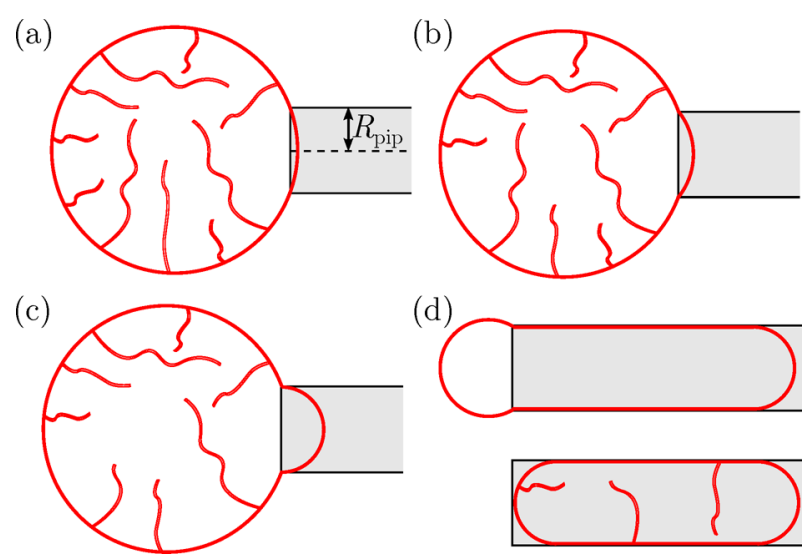

Figure 1. Different stages for the aspiration process of a tubulated GUV (red) by a micropipette (gray) of radius $R_{\text {pip. }}$ (a) Spherical mother vesicle comes into contact with the pipette. (b) With increasing suction pressure, some of the nanotubes are retracted, and the mother vesicle develops a tongue that has the form of a spherical cap. (c) When the suction pressure reaches a critical value, the cap-like tongue becomes a hemisphere with radius $R_{\text {pip }}$, and the vesicle membrane starts to flow into the micropipette. (d) Depending on the membrane area stored in the nanotubes, the vesicle motion stops as soon as all nanotubes have been retracted (d, top) or continues until the vesicle is completely aspirated into the pipette ( $d$, bottom). directly measured here by analyzing the evolution of the vesicle shape during initial aspiration. Our experimental data confirm the theoretical prediction ${ }^{22}$ that the presence of membrane nanotubes leads to a mechanical tension that is several orders of magnitude smaller than the spontaneous tension. Indeed, for the bilayer membranes studied here, the spontaneous tension is on the order of $10 \mathrm{pN} / \mu \mathrm{m}$ or $10^{-2} \mathrm{mN} / \mathrm{m}$, whereas the absolute value of the mechanical tension is smaller by 2 orders of magnitude. In this way, our results provide insight into the intricate and composite nature of membrane tension. Because of their increased robustness, tubulated GUVs represent improved research tools for membrane science, in general, with potential applications as storage and delivery systems as well as cell-like microcompartments in bioengineering, pharmacology, and synthetic biology.

\section{RESULTS AND DISCUSSION}

Spontaneous Tubulation of Giant Vesicles Doped with GM1. We prepared GUVs, composed of the phospholipid POPC, a few mol \% of the glycolipid (or ganglioside) GM1, and $0.1 \mathrm{~mol} \%$ of the fluorescently labeled lipid Texas-RedDHPE (dihexadecanoylglycerophosphoethanolamine) by electroformation $^{28}$ as described in the Methods section. The same preparation method has been previously used in ref 29. However, in contrast to the latter study, we subsequently diluted the vesicle suspension ten- to eleven-fold with native buffer, which led to the formation of stable nanotubes inside the GUVs, reflecting the larger GM1 concentration within the inner bilayer leaflets. This bilayer asymmetry is caused by the desorption of the GM1 molecules from the outer leaflets into the exterior solution, which is depleted of GM1 molecules because of the dilution step. After tubulation, the excess membrane area stored in the tubes was typically between 20 and $50 \%$ of the total membrane area. At room temperature, the binary lipid mixture of POPC and GM1 undergoes phase separation when the mole fraction of GM1 exceeds about 5 mol $\% .{ }^{29}$ Here, we focused on lipid compositions with 2 and 4 mol $\%$ of GM1 for which we did not detect any intramembrane domains.

Examples for tubulated vesicles with these two lipid compositions are displayed in Figure 2. The nanotubes were only visible in the presence of the fluorescently labeled lipids, in
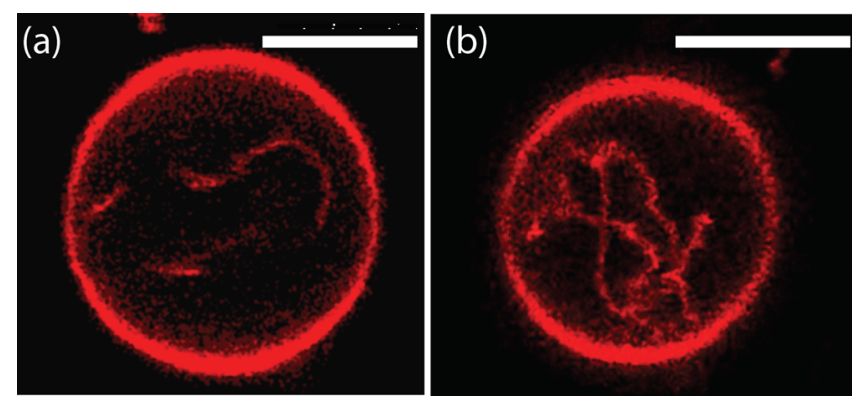

Figure 2. Confocal cross sections of membrane nanotubes protruding from the mother vesicles (large red circles) into the vesicle interior. The vesicle membranes consist of the phospholipid POPC doped with the fluorescently labeled lipid Texas-Red-DHPE as well as $2 \mathrm{~mol} \%$ of GM1 in (a) and $4 \mathrm{~mol} \%$ of GM1 in (b). The nanotubes are only visible in the presence of the fluorescently labeled lipids (red), in agreement with the theoretical analysis which implies that the nanotubes have a width on the order of 100 nm. Scale bars: $10 \mu \mathrm{m}$. 
agreement with the theoretical analysis described below, which implies that the nanotubes have a width on the order of 100 $\mathrm{nm}$, that is, below optical resolution. For the vesicle in Figure $2 \mathrm{~b}$, a three-dimensional stack of confocal $x y$ cross sections is displayed in Movie 1 of the Supporting Information (SI), which reveals the three-dimensional conformations of the nanotubes. Inspection of this movie shows that these tubes are quite long and attain complex and highly curved conformations. Below, we will compare this experimentally observed flexibility with theoretical estimates for the persistence length of the tubes. This comparison indicates that the tubes have a necklace-like morphology.

We also prepared vesicles with nanotubes pointing toward the exterior solution by adding the GM1 molecules to the GUV dispersion without the dilution step (see Figure 3). In the
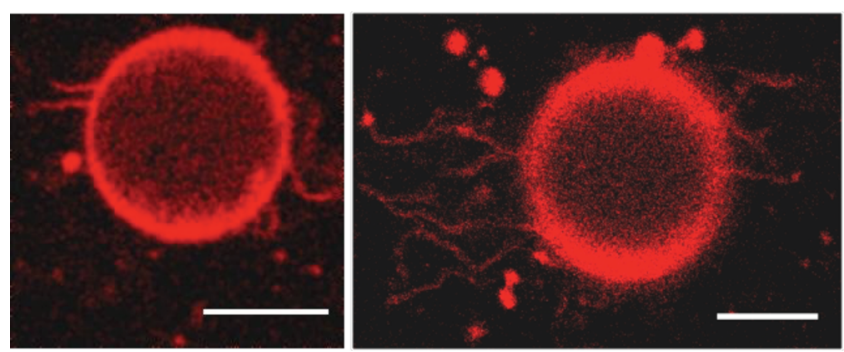

Figure 3. Confocal cross sections of membrane nanotubes protruding from the mother vesicles (red circles) toward the exterior solution. Confocal cross section of two GUVs prepared from POPC and $4 \mathrm{~mol} \%$ of GM1 that formed out-tubes after the addition of GM1 to the vesicle suspension. The out-tubes have a width below the optical resolution and are labeled by the same fluorophore (red) as in Figure 2. Scale bars: $10 \mu \mathrm{m}$.

presence of such out-tubes, the vesicles have a fuzzy surface and an ill-defined size. Thus, in the following, we will focus on GUVs with in-tubes that have a smooth surface and a welldefined size as in Figure 2.

The latter GUVs were aspirated by micropipettes (see the Methods section) in order to expose them to a tunable mechanical tension and to retract the tubes in a controlled and reversible manner. During initial aspiration, as shown in Figure $1 \mathrm{a}-\mathrm{c}$ and Figure $4 \mathrm{a}, \mathrm{b}$, the emerging membrane tongue forms a spherical cap with a radius that decreases from the initial radius of the mother vesicle to the radius of the micropipette. The quantitative analysis of the shape evolution during the initial aspiration process allows us to directly measure the spontaneous tension of the GUV membrane, which is intimately related to the bilayer asymmetry and the associated spontaneous curvature of this membrane. To the best of our knowledge, initial aspiration of GUVs by micropipettes has not been studied previously in a systematic and quantitative manner.

Initial Aspiration: Theory and Experiment. In the spontaneous curvature model, ${ }^{30,31}$ the vesicle membrane is described by two fluid-elastic parameters, the spontaneous curvature $m$ and the bending rigidity $\kappa$. These two parameters can be combined into an intrinsic tension scale, the spontaneous tension $\sigma \equiv 2 \mathrm{\kappa m}^{2}$, which varies quadratically with the spontaneous curvature $m$ and represents the bending energy density of a weakly curved membrane segment that has a relatively large spontaneous curvature but cannot adapt its local mean curvature to this spontaneous curvature. ${ }^{22}$ The total membrane tension $\hat{\Sigma}$ can then be decomposed according to

$$
\hat{\Sigma}=\Sigma+\sigma=\Sigma+2 \kappa m^{2}
$$

where the mechanical tension $\Sigma$ depends, in general, on the size and shape of the vesicle as well as on external constraints and forces acting on the vesicle. As shown in ref 22, the spontaneous curvature model ${ }^{30,31}$ leads to a Euler-Lagrange or shape equation that depends explicitly on the total membrane tension $\hat{\Sigma}$, which is equal to the superposition of the mechanical and the spontaneous tension as in eq 1 . In the SI, we utilize this shape equation for spherical and cylindrical membrane segments to derive explicit expressions for the mechanical tension of the tubulated GUVs.

Let us now consider a giant vesicle with nanotubes protruding into the vesicle interior, as shown in Figure 2. The presence of the nanotubes reveals a large bilayer asymmetry or spontaneous curvature $m$. Because the nanotubes point toward the vesicle interior, the spontaneous curvature $m$ is negative, and the tube radius $R_{\mathrm{nt}}$ is equal to $1 /(2 \mid \mathrm{ml})$ or $1 /|\mathrm{m}|$ depending on whether the tubes are cylindrical or necklacelike. ${ }^{2,22}$ Tubulated GUVs are characterized by a large separation of length scales: the spherical mother vesicle has a radius $R_{\mathrm{mv}}$ on the order of $10 \mu \mathrm{m}$, whereas the nanotubes have a radius $R_{\mathrm{nt}}$ that is close to or below optical resolution. For the vesicles
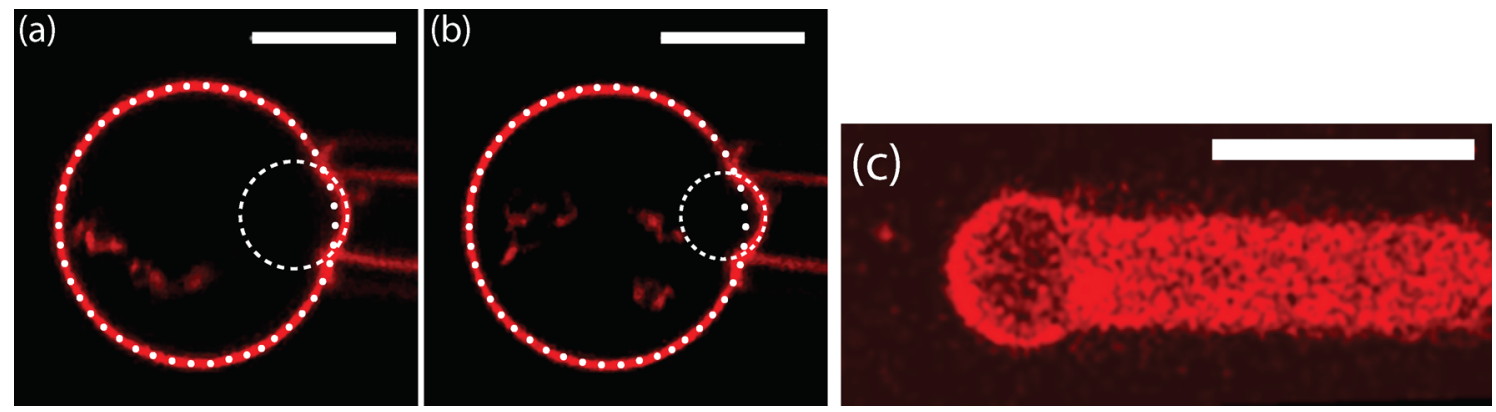

Figure 4. Shape evolution of aspirated GUV as observed with fluorescence microscopy. (a,b) Initial aspiration of a tubulated vesicle composed of POPC and $4 \mathrm{~mol} \%$ of GM1, with $0.1 \mathrm{~mol} \%$ of the fluorescently labeled lipid Texas-Red-DHPE (red). (a) Mother vesicle (large dotted circle) has the radius $R_{\mathrm{mv}}$ and forms a small tongue that has the shape of a spherical cap (small dashed circle). The radius $R_{\mathrm{to}}$ of this cap is smaller than $R_{\mathrm{mv}}$ and larger than the pipette radius $R_{\text {pip. }}$ (b) When the suction pressure reaches a critical value, the tongue attains a hemispherical shape with radius $R_{\mathrm{to}}=R_{\text {pip }}$, and the vesicle membrane starts to flow into the pipette. (c) Snapshot of a flowing vesicle just before it is completely aspirated into the pipette. The detailed time evolution of this latter process is displayed in Movie 2 of the SI. Scale bars in $(\mathrm{a}-\mathrm{c}): 10 \mu \mathrm{m}$. 
studied here, the tube radius $R_{\mathrm{nt}}$ was on the order of $100 \mathrm{~nm}$ and, thus, two orders of magnitude smaller than the radius $R_{\mathrm{mv}}$ of the mother vesicle, with the ratio $R_{\mathrm{nt}} / R_{\mathrm{mv}} \lesssim 0.01$. Furthermore, the length of the nanotubes was much larger than the tube width. This separation of length scales implies that the absolute value $|\Sigma|$ of the mechanical tension behaves as

$$
|\Sigma| \simeq \frac{R_{\mathrm{nt}}}{R_{\mathrm{mv}}} \sigma \ll \sigma=2 \kappa \mathrm{m}^{2} \text { for } R_{\mathrm{mv}} \gg R_{\mathrm{nt}} \simeq \frac{1}{|\mathrm{~m}|}
$$

and is therefore several orders of magnitude smaller than the spontaneous tension. As a consequence, we can ignore the mechanical tension, which depends on the size and shape of the GUV, compared to the spontaneous tension, which represents a curvature-elastic material parameter. The inequality in eq 2 , which follows from the mechanical equilibrium between the highly curved nanotubes and the weakly curved membrane of the mother vesicle, has been previously derived for cylindrical nanotubes. $^{22}$ As shown in the SI, the same inequality applies to necklace-like nanotubes, as well.

During the initial aspiration of the tubulated vesicle, the mother vesicle forms a membrane tongue that has the form of a small spherical cap with curvature radius $R_{\text {to }}$ (see Figure $1 \mathrm{~b}$ and Figure $4 a$ ). For such a piece-wise spherical shape, minimization of the membrane's bending energy leads to the pressure balance

$$
\Delta P \equiv P_{\mathrm{ex}}-P_{\text {pip }}=P_{\mathrm{el}}\left(R_{\mathrm{to}}, R_{\mathrm{mv}}\right)
$$

between $(i)$ the suction pressure $\Delta P$, which depends on the pressures $P_{\text {ex }}$ and $P_{\text {pip }}$ in the exterior aqueous solution and in the pipette, respectively, and (ii) the elastic counter pressure

$$
P_{\mathrm{el}}(x, y) \equiv\left(\frac{1}{x}-\frac{1}{y}\right)\left[2 \Sigma+2 \sigma-4 \kappa m\left(\frac{1}{x}+\frac{1}{y}\right)\right]
$$

which depends on the radii $x=R_{\mathrm{to}}$ and $y=R_{\mathrm{mv}}$ and, thus, on the vesicle geometry. The relationships 3 and 4 are explicitly derived in the SI. These relationships describe the mechanical balance between the suction pressure $\Delta P$, which represents an external control parameter, and the counter pressure $P_{\mathrm{el}}$ arising from the elasticity of the membrane. A suction pressure $\Delta P>0$ pulls the vesicle into the pipette, whereas a counter pressure $P_{\mathrm{el}}$ $>0$ pushes the vesicle out of the pipette.

It follows from eq 4 that the elastic counter pressure $P_{\mathrm{el}}$ depends, in general, both on the mechanical tension $\Sigma$ that stretches the membrane and on the spontaneous tension $\sigma$ that increases the bending energy of the weakly curved membrane segments. However, for the tubulated vesicles considered here, the mechanical tension can be ignored compared to the spontaneous tension, as shown in the SI and summarized by the relation 2. Therefore, the elastic counter pressure as given by eq 4 has the asymptotic form

$$
P_{\mathrm{el}}\left(R_{\mathrm{to}}, R_{\mathrm{mv}}\right) \approx 2 \sigma\left(\frac{1}{R_{\mathrm{to}}}-\frac{1}{R_{\mathrm{mv}}}\right) \text { for } R_{\mathrm{to}} \gg \frac{1}{|m|}
$$

An analogous equation applies to the micropipette aspiration of liquid droplets, ${ }^{32-34}$ for which the counter pressure arises from capillary forces, and the spontaneous tension $\sigma$ of the GUV is replaced by the interfacial tension of the droplet.

In the micropipette experiments, the vesicles were aspirated by a tunable suction pressure using the hydraulic pressure system described in the Methods section. The initial aspiration regime could be explored by increasing the suction pressure up to $3 \mathrm{~Pa}$ (see Figure 5). Using epifluorescence microscopy, we

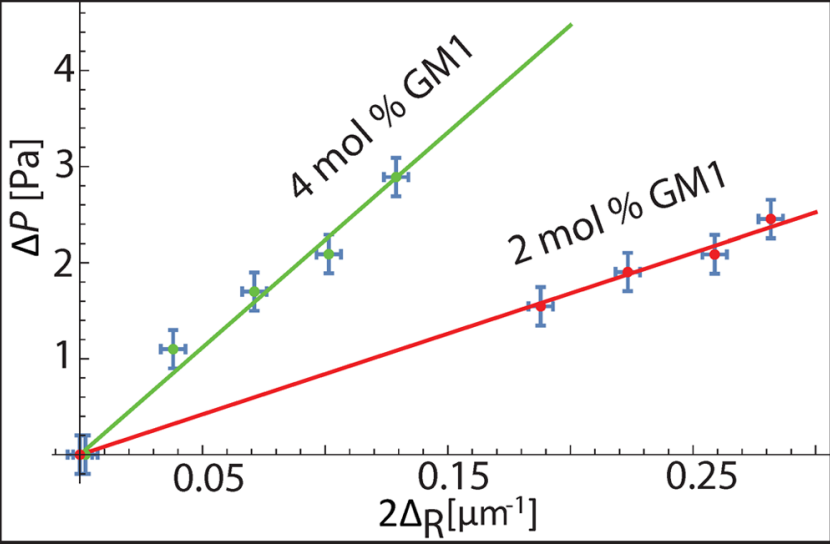

Figure 5. Direct measurement of spontaneous tension. Suction pressure $\Delta P=P_{\mathrm{ex}}-P_{\mathrm{pip}}$ versus twice the inverse length $\Delta_{R}=1 / R_{\text {to }}$ $-1 / R_{\mathrm{mv}}$ as defined in eq 6 , for 2 and $4 \mathrm{~mol} \%$ of GM1. The radius $R_{\text {to }}$ of the emerging tongue and the radius $R_{\mathrm{mv}}$ of the spherical mother vesicle were directly obtained from the optical micrographs. The data are in good agreement with the linear relation $\Delta P$ $=P_{\mathrm{el}} \approx 2 \sigma \Delta_{R}$ as given by eqs 3 and 5 , leading to the spontaneous tension $\sigma=8.33 \pm 0.76$ and $\sigma=22.2 \pm 1.5 \mathrm{~Pa} \mu \mathrm{m}$ for 2 and $4 \mathrm{~mol}$ $\%$ of GM1, respectively.

measured the radius $R_{\mathrm{to}}$ of the emerging tongue and the radius $R_{\mathrm{mv}}$ of the mother vesicle. In Figure 5, we plot the suction pressure $\Delta P$ as a function of the inverse length

$$
\Delta_{R} \equiv \frac{1}{R_{\mathrm{to}}}-\frac{1}{R_{\mathrm{mv}}}
$$

for two different values of the overall GM1 concentration. Fitting the experimental data in Figure 5 to the pressure balance in eq 3 with the counter pressure $P_{\mathrm{el}}$ as given by eq 5 , we obtain the spontaneous tensions $\sigma=8.33 \pm 0.76$ and $22.2 \pm$ $1.5 \mathrm{pN} / \mu \mathrm{m}$ for 2 and $4 \mathrm{~mol} \%$ of GM1, respectively. The values of the spontaneous curvature $m$ then follow from $m=-\sqrt{\sigma /(2 \kappa)}$, where the minus sign takes into account that the GUVs studied here formed in-tubes.

The bending rigidity $\kappa$ of POPC membranes doped with GM1 has been previously measured in ref 29. In the latter study, the GUVs were prepared without the additional dilution step and thus without the GM1 desorption from the outer leaflet that acts to reduce the final GM1 concentration in the membranes. In the regime of low GM1 fractions, the bending rigidity $\kappa$ was found to be on the order of $10^{-19} \mathrm{~J}$. When we combine this $\kappa$ value with the measured values of the spontaneous tension $\sigma$, the relation $m=-\sqrt{\sigma /(2 \kappa)}$ leads to the spontaneous curvatures $m \simeq-1 /(155 \mathrm{~nm})$ and $m \simeq-1 /$ $(95 \mathrm{~nm})$ for 2 and $4 \mathrm{~mol} \%$ of GM1, with an accuracy of about $\pm 20 \%$. In order to adapt their shape to these spontaneous curvatures, the membranes form necklace-like or cylindrical nanotubes with a radius of $1 /|\mathrm{m}|$ or $1 /(2|\mathrm{~m}|)$, respectively. Therefore, we conclude that the tubes displayed in Figure 2 have a diameter below or close to optical resolution for both 2 and $4 \mathrm{~mol} \%$ of GM1.

The deduced values of the spontaneous curvatures allow us to distinguish necklace-like and cylindrical nanotubes by estimating the persistence lengths of both types of tubes and then comparing these estimates with the thermally excited undulations of the tubes as observed experimentally. As shown in ref 2 , the persistence length of a cylindrical nanotube is $\pi \kappa /(\mathrm{I}$ 

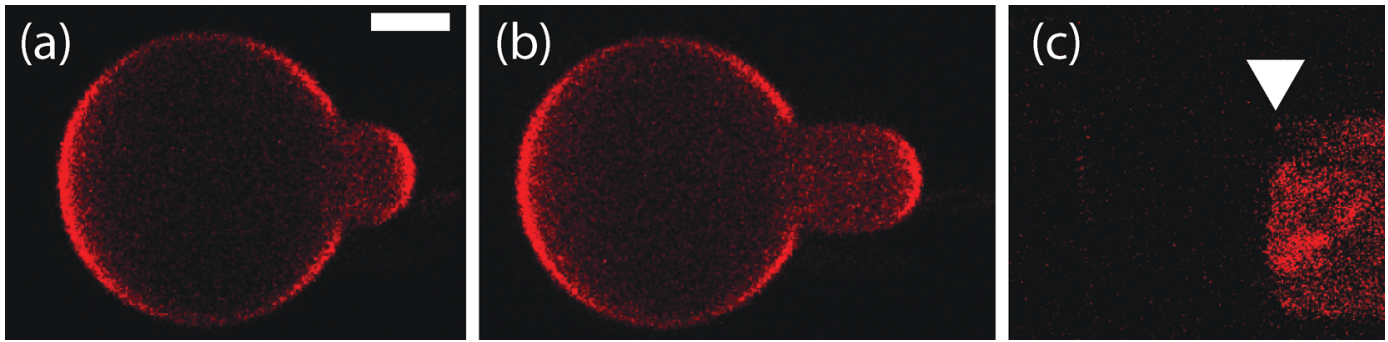

Figure 6. Rupture of GUV in the absence of nanotubes. (a,b) When a GUV without nanotubes as formed in the absence of GM1 is aspirated into the micropipette, the mechanical tension arising from the suction pressure stretches the GUV membrane (red) until the membrane ruptures and the GUV bursts. The latter process leads to lipid coating of the pipette as shown in (c). The arrowhead indicates the location of the pipette tip. The suction pressure increases continuously during the whole aspiration process until rupture, and the system does not exhibit a critical pressure at which the vesicle starts to flow into the pipette. The scale bar is $10 \mu \mathrm{m}$ and applies to all three images.

$\left.m \mid k_{\mathrm{B}} T\right)$, whereas the same length of a necklace-like tube is expected to be on the order of $2 / \mid \mathrm{ml}$. For a bending rigidity $\kappa \simeq$ $10^{-19}$ and a spontaneous curvature of about $1 /(100 \mathrm{~nm})$, we then obtain a persistence length of about $200 \mathrm{~nm}$ and $6 \mu \mathrm{m}$ for necklace-like and cylindrical nanotubes, respectively. Inspection of Figure 2 and Movie 1 reveals many sharp bends on the micrometer scale, which indicates that the tubes are necklacelike.

Critical Suction Pressure and Mechanical Instability. The initial aspiration process continues until the vesicle tongue attains a hemispherical shape. For this shape, the tongue radius $R_{\text {to }}$ is equal to the pipette radius $R_{\text {pip }}$, and the mother vesicle has a certain size $R_{\mathrm{mv}}=R_{\mathrm{mv}}^{*}$. The pressure balance then becomes

$$
\Delta P=P_{\mathrm{el}}^{*} \equiv P_{\mathrm{el}}\left(R_{\mathrm{pip}}, R_{\mathrm{mv}}^{*}\right)
$$

which defines the critical pressure $P_{\mathrm{el}}^{*}$. The latter pressure has the simple form

$$
P_{\mathrm{el}}^{*} \approx 2 \sigma\left(\frac{1}{R_{\mathrm{pip}}}-\frac{1}{R_{\mathrm{mv}}^{*}}\right) \text { for } R_{\text {pip }} \gg \frac{1}{|m|}
$$

The radius $R_{\mathrm{mv}}^{*}$ of the mother vesicle at the critical pressure depends primarily on the initial size of the vesicle and on the pipette radius.

The pressure balance between the suction pressure and the elastic counter pressure as described by eq 3 leads to a sequence of stable equilibrium states during initial aspiration, that is, as long as $R_{\text {to }}>R_{\text {pip }}$ and $\Delta P<P_{\text {el }}^{*}$. Thus, consider a state of mechanical equilibrium with $\Delta P=P_{\mathrm{el}}\left(R_{\mathrm{to}}, R_{\mathrm{mv}}\right)<P_{\mathrm{el}} *$ and a perturbation of this state that moves the tongue further into the pipette, thereby reducing the tongue radius to $R_{\mathrm{to}}{ }^{\prime}<$ $R_{\text {to }}$ for fixed suction pressure $\Delta P$. The resulting counter pressure $P_{\mathrm{el}}\left(R_{\mathrm{to}}{ }^{\prime}\right)$ exceeds the equilibrium value $P_{\mathrm{el}}\left(R_{\mathrm{to}}\right)=\Delta P$ as follows from eq 5 , and the positive pressure difference $P_{\mathrm{el}}\left(R_{\mathrm{to}}{ }^{\prime}\right)-\Delta P$ pushes the tongue back toward its equilibrium state.

In contrast, a perturbation that moves the tongue further into the pipette for the critical suction pressure $\Delta P=P_{\mathrm{el}}^{*}$ now leads to a smaller radius $R_{\mathrm{mv}}{ }^{\prime}<R_{\mathrm{mv}}^{*}$ of the mother vesicle, whereas the tongue radius $R_{\mathrm{to}}=R_{\text {pip }}$ remains unchanged. It then follows from eq 5 that the counter pressure $P_{\mathrm{el}}\left(R_{\mathrm{mv}}{ }^{\prime}\right)$ is smaller than the suction pressure $\Delta P$, which implies that the tongue is pushed even further into the pipette. As a consequence, as soon as the suction pressure has reached the critical value $P_{\mathrm{el}}^{*}$, the vesicle membrane starts to flow into the micropipette, as observed experimentally; see Movie 2 in the SI. This movie displays a sequence of confocal $x y$ cross sections of the flowing vesicle which was kept at constant aspiration pressure until the vesicle was completely sucked up and disappeared into the pipette, as visible in the last frame of Movie 2.

Essentially the same mechanical instability has been observed for liquid droplets, ${ }^{32-34}$ neutrophils (i.e., certain types of white blood cells), ${ }^{3,35}$ and fibroblasts. ${ }^{36}$ For liquid droplets, the parameter that replaces the spontaneous tension $\sigma$ in expression 5 for the elastic counter pressure is the interfacial tension of the liquid-liquid interface. For neutrophils, the corresponding parameter was proposed to be the cortical tension generated by the actomyosin cortex. For fibroblasts, this parameter was decomposed into the cortical tension, and another tension that remains after the cortex has been disassembled chemically by cytochalasin $\mathrm{D} .{ }^{36}$ It is interesting to note that the membrane tension measured in ref 36 after the disassembly of the actin cortex is on the same order of magnitude as the spontaneous tensions obtained here.

The membrane flow arising from the mechanical instability of tubulated vesicles can lead to two different final states, depending on the membrane area initially stored in the nanotubes. If this area is relatively small, all of these tubes will be retracted before the whole vesicle has been sucked into the pipette, and the vesicle reaches a new equilibrium state, as shown by the top cartoon in Figure 1d. Further aspiration then leads to membrane stretching until membrane rupture and vesicle bursting, as demonstrated in Figure 6 for a GUV membrane without GM1.

On the other hand, if the membrane area stored in the tubes is sufficiently large, the vesicle will be aspirated completely into the micropipette with some remaining area stored in tubes, see Figure 1d, bottom and Movie 2 in the SI. The tube retraction is completely reversible: as long as the vesicle has not been sucked up completely by the micropipette, we can recover the nanotubes by reversing the changes in aspiration pressure at each stage of the aspiration process. In addition, the tubulated GUVs are sufficiently robust to tolerate repeated cycles of tube retraction coupled to in-flow of the membrane tongue and subsequent tube elongation coupled to out-flow of this tongue (see Movie 3 in the SI). The latter movie displays the response of one tubulated GUV exposed to several changes in the suction pressure and represents a sequence of five subsequent recordings including three full cycles of strong aspiration into the micropipette and subsequent release from this pipette. The total recording time of Movie 3 exceeded $1 \mathrm{~h}$. On this time scale, we did not detect any significant changes in the behavior of the tubes, which indicates that the bilayer asymmetry and the associated spontaneous tension of the GUV membrane were not affected by the recurrent mechanical stress generated by the 

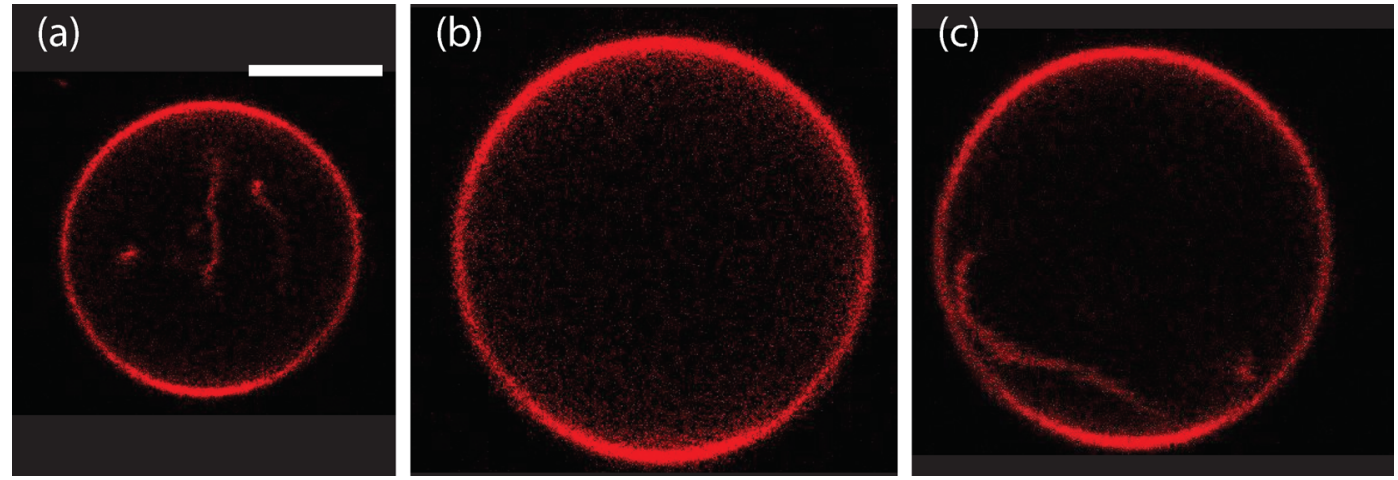

Figure 7. Robustness of tubulated GUVs against changes in the osmotic conditions. Equatorial cross sections of the same vesicle (a) before osmotic inflation, (b) after inflation, and (c) after subsequent deflation. In (a), the vesicle was exposed to an exterior buffer with $2 \mathrm{mM}$ HEPES. The inflation from (a) to (b) and the deflation from (b) to (c) were obtained by adding buffers with 1 and 2 mM HEPES to the exterior solution, respectively. Essentially all tubes have been retracted in (b), but new tubes start to form in (c). The scale bar is $10 \mu \mathrm{m}$ and applies to all three images. The different sizes of the mother vesicle (red circle) in (a) and (b) directly reveal the large membrane area stored in the nanotubes.

micropipette. Another example for the increased robustness of tubulated vesicles is provided by their response to changes in the osmotic conditions, as illustrated in Figure 7. In this figure, a tubulated vesicle is first inflated osmotically until essentially all nanotubes have been retracted. When the latter vesicle is subsequently deflated, the resulting membrane excess area is again stored in membrane nanotubes.

\section{CONCLUSIONS}

In this paper, we introduced GUVs with spontaneously formed membrane nanotubes as model systems that mimic cellular membranes with reservoirs for membrane area and demonstrated that these systems resist even strong mechanical perturbations without rupture. A quantitative comparison between theory and experiment revealed that tubulated GUVs respond to mechanical perturbations in close analogy to liquid droplets, with the spontaneous tension of the membranes playing the same role for tubulated GUVs as the interfacial tension for liquid droplets. In fact, the spontaneous tension $\sigma=2 \mathrm{~km}^{2}$ represents a curvature-elastic material parameter that determines both the initial aspiration process (see eqs 3-5) and the critical suction pressure as given by eq 8 . For the lipid compositions studied here, the numerical values of the spontaneous tension $\sigma$ have been determined from the shape evolution of the membrane tongue during initial aspiration (Figure 4a,b and Figure 5). To the best of our knowledge, this initial aspiration process has not been studied previously in a systematic and quantitative manner. When we increase the GM1 concentration from 2 to $4 \mathrm{~mol} \%$, the spontaneous tension is increased from $\sigma \simeq 8.3$ to $22 \mathrm{pN} / \mu \mathrm{m}$, and the spontaneous curvature from $m \simeq-1 /(155 \mathrm{~nm})$ to -1 / $(95 \mathrm{~nm})$, respectively.

In the present study, we used a specific lipid composition to produce giant vesicles with nanotubes, but such vesicles can also be produced for other types of lipid compositions. One example is provided by ternary lipid mixtures of DOPC, DPPC, and cholesterol which form nanotubes both in their liquidordered and in their liquid-disordered phase when the two leaflets of the membranes are exposed to different PEG concentrations. ${ }^{2}$ In fact, any lipid membrane with a sufficiently strong bilayer asymmetry can form such tubes. We demonstrated the increased robustness of tubulated GUVs by micropipette aspiration with a tunable suction pressure (Figure
3 and Movie 2 in the SI) and by changes in the osmotic conditions (Figure 7). The micropipette experiments directly imply that GUVs with nanotubes can pass through small blood vessels or microfluidic channels without rupture even if the diameter of these vessels and channels is much smaller than the vesicle size. In the latter context, the tubulated vesicles could be used as storage and delivery systems. One example is provided by nonadhesive nanoparticles that are confined within necklacelike nanotubes. Tube retraction in response to external perturbations will then lead to the partial release of the stored particles. Another example is the enrichment of adhesive Janus particles within the nanotubes as a result of curvature-induced forces that move such particles toward the highly curved tubes after they have been adsorbed onto the weakly curved mother vesicle. $^{37}$

It is instructive to compare the approach described here, based on tubulated GUVs, with other methods that have been developed to increase the stability and robustness of giant vesicles. To increase the rupture (or lysis) tension of lipid bilayers, two classical approaches are to use polymerizable lipids $^{38}$ or to add additional molecular components such as cholesterol. $^{39}$ One may also replace the lipids by diblock copolymers to form polymeric GUVs or polymersomes, ${ }^{40,41}$ which have an increased rupture tension, as well. ${ }^{42}$ In the context of whole-GUV patch clamping, the contact area between the giant vesicle and the inner surface of the micropipette can be stabilized by $\beta$-casein that prevents the vesicle membrane from spreading further into the pipette. ${ }^{43} \mathrm{~A}$ general strategy to mechanically stabilize GUVs that has been introduced quite recently is to assemble these vesicles within microfluidic emulsion droplets and to use the droplet surfaces as mechanical scaffolds for the vesicles. ${ }^{10}$ Compared to these alternative strategies, the tubulated GUVs described here have a number of significant advantages: (i) The lipid membranes considered here exhibit the usual fluidity of phospholipid membranes, which mimics the fluidity of cellular membranes. (ii) These lipid membranes are biocompatible and provide the most natural environment for membrane proteins. (iii) The tubulated GUVs are fully immersed in water and are accessible to solutes and biomolecules from both the exterior and the interior aqueous solution. (iv) The increased robustness of tubulated GUVs arises from internal membrane reservoirs, in close analogy to cellular membranes, which implies that these 
giant vesicles exhibit cell-like responses to mechanical perturbations.

Indeed, giant vesicles with membrane nanotubes will also tolerate other mechanical perturbations, arising, for example, from the adhesion and engulfment of large bodies, in close analogy to the cellular uptake via phagocytosis and pinocytosis, or in response to constriction forces that can lead to fission of the GUVs into smaller membrane compartments. The latter process of artificial cytokinesis is an important objective for the bottom-up assembly of artificial protocells., ${ }^{9,}$ In general, tubulated GUVs, which are characterized by very low values of the mechanical tension, represent improved research tools for membrane science with potential applications as storage and delivery systems as well as cell-like microcompartments in bioengineering, pharmacology, and synthetic biology.

\section{METHODS}

Vesicle Preparation. GUVs were grown using the electroformation technique. ${ }^{1,28}$ 1-Palmitoyl-2-oleoyl-sn-glycero-3-phosphatidylcholine was purchased from Avanti Polar Lipids (dissolved in a chloroform solution to a concentration of $10 \mathrm{mg} / \mathrm{mL}$ ). GM1 was purchased from Avanti Polar Lipids and dissolved in a dichloromethane/methanol (2:1) solution to a concentration of $3 \mathrm{mM}$. Two samples were prepared: (i) $3 \mathrm{mM}$ POPC with $2 \mathrm{~mol} \%$ of GM1 and (ii) $3 \mathrm{mM}$ POPC with $4 \mathrm{~mol} \%$ of GM1. For fluorescence imaging of the vesicles, additionally $0.1 \mathrm{~mol} \%$ of Texas-Red-DHPE was added to samples 1 and 2. Two indium-tin oxide (ITO)-coated glass plates (Delta Technologies Ltd.) were preheated at $55^{\circ} \mathrm{C}$ for $5-10 \mathrm{~min}$, and an aliquot of $10 \mu \mathrm{L}$ of the sample solution was spread onto the electrically conductive sides of the ITO plates. The ITO slides were dried under vacuum at $55^{\circ} \mathrm{C}$ for $2 \mathrm{~h}$ to remove the organic solvents. They were put against a $2 \mathrm{~mm}$ thick Teflon spacer to form a closed chamber. HEPES buffer ( $1 \mathrm{mM}, \mathrm{pH} 7.4,0.5 \mathrm{Na}$ HEPES; SigmaAldrich, St. Louis, MO) was preheated at $55{ }^{\circ} \mathrm{C}$ and filled into the chamber ( 2 mM HEPES buffer was used in the experiments displayed in Figure 7). A sinusoidal AC electric field at $10 \mathrm{~Hz}$ was applied for electroswelling the lipid films at $55{ }^{\circ} \mathrm{C}$. In the first phase of the electroswelling process, the amplitude of the applied field was linearly increased from $0.1 \mathrm{~V}$ (peak to peak) to $0.8 \mathrm{~V}$ (peak to peak) over 30 min. Thereafter, the amplitude of the AC field was kept constant during 60-120 $\mathrm{min}$ to grow the vesicles. Finally, during the last 60 min, the GUVs were separated from the ITO surface by linearly decreasing the voltage and frequency to $0.5 \mathrm{~V}$ and $1 \mathrm{~Hz}$. After electroswelling, GUVs were cooled to $23{ }^{\circ} \mathrm{C}$ in an oven at a rate of 0.1 ${ }^{\circ} \mathrm{C} / \mathrm{min}$. The resulting vesicles were found to be stable and could be transferred to an observation chamber for optical investigations. The latter chamber was closed using sealing paste (Korasilon from Carl Roth, \#0856.1) to stop fluid flow and evaporation.

Formation of Out-Tubes after External Addition of GM1. GM1 was dissolved in a dichloromethane/methanol (2:1) solution to a concentration of $3 \mathrm{mM}$. The organic solvent was removed under vacuum at $55{ }^{\circ} \mathrm{C}$. Milli-Q water was added to reach a GM1 concentration of $3 \mathrm{mM}$, and the sample was stirred. One microliter of this GM1 stock solution was added to $500 \mu \mathrm{L}$ of GUV suspension to increase the concentration of GM1 in the outer leaflet by spontaneous insertion of the molecules from the surrounding concentrations. The chamber was closed with the same sealing paste. Immediately afterward, the vesicles were observed to exhibit out-tubes as in Figure 3.

Micropipette Aspiration System. Micropipettes were prepared from glass capillaries (World Precision Instruments Inc.) that were pulled using a pipette puller (Sutter Instruments, Novato, CA). Pipette tips were cut using a microforge (Narishige, Tokyo, Japan) to obtain tips with an inner diameter between 4 and $6 \mu \mathrm{m}$. Adhesion of the membrane to the pipette was prevented by incubation of the pipette tips in $1 \mathrm{mg} / \mathrm{mL}$ aqueous solution of casein (Sigma). A new pipette was used for the aspiration of each GUV. After the pipette was inserted into the observation chamber, the zero pressure across the pipette tip was attained and calibrated by watching the flow of small particles within the tip. The aspiration pressure was controlled through adjustments in the height of a reservoir mounted on a linear translational stage (M-531.PD; Physik Instrumente, Germany); for further details see the Supporting Information of ref 44. This setup allowed the pressure to increase up to $2 \mathrm{kPa}$ with a pressure resolution of $1 \mathrm{mPa}$. The pressure was changed by displacing the water reservoir at a speed of $0.01 \mathrm{~mm} / \mathrm{s}$. The displacement was then stopped for 2-3 min before any data were recorded.

\section{ASSOCIATED CONTENT}

\section{S Supporting Information}

The Supporting Information is available free of charge on the ACS Publications website at DOI: 10.1021/acsnano.8b00640.

Supplementary text with derivations of eqs 2-4 (PDF) Movie captions (PDF)

Movie 1 (MPG)

Movie 2 (MPG)

Movie 3 (AVI)

\section{AUTHOR INFORMATION}

\section{Corresponding Author}

*E-mail: lipowsky@mpikg.mpg.de.

ORCID $\odot$

Reinhard Lipowsky: 0000-0001-8417-8567

Notes

The authors declare no competing financial interest.

\section{ACKNOWLEDGMENTS}

We acknowledge support by the German Research Foundation (DFG) via the IRTG 1524 as well as by the Max Planck Society and the Federal Ministry of Education and Research (BMBF) via the MaxSynBio consortium.

\section{REFERENCES}

(1) Dimova, R.; Aranda, S.; Bezlyepkina, N.; Nikolov, V.; Riske, K.; Lipowsky, R. A Practical Guide to Giant Vesicles: Probing the Membrane Nanoregime via Optical Microscopy. J. Phys.: Condens. Matter 2006, 18, S1151-S1176.

(2) Liu, Y.; Agudo-Canalejo, J.; Grafmüller, A.; Dimova, R.; Lipowsky, R. Patterns of Flexible Nanotubes Formed by LiquidOrdered and Liquid-Disordered Membranes. ACS Nano 2016, 10, 463-474.

(3) Pezeshkian, W.; Gao, H.; Arumugam, S.; Becken, U.; Bassereau, P.; Florent, J.-C.; Ipsen, J. H.; Johannes, L.; Shillcock, J. C. Mechanism of Shiga Toxin Clustering on Membranes. ACS Nano 2017, 11, 314324.

(4) Schmid, E. M.; Bakalar, M. H.; Choudhuri, K.; Weichsel, J.; Ann, H. S.; Geissler, P. L.; Dustin, M. L.; Fletcher, D. A. Size-Dependent Protein Segregation at Membrane Interfaces. Nat. Phys. 2016, 12, 704-711.

(5) Chen, Z.; Zhu, C.; Kuo, C. J.; Robustelli, J.; Baumgart, T. The NTerminal Amphipathic Helix of Endophilin Does Not Contribute to Its Molecular Curvature Generation Capacity. J. Am. Chem. Soc. 2016, $138,14616-14622$

(6) Talbot, E. L.; Parolini, L.; Kotar, J.; Di Michele, L. D.; Cicuta, P. Thermal-Driven Domain and Cargo Transport in Lipid Membranes. Proc. Natl. Acad. Sci. U. S. A. 2017, 114, 846-851.

(7) Snead, W. T.; Hayden, C. C.; Gadok, A. K.; Zhao, C.; Lafer, E. M.; Rangamani, P.; Stachowiak, J. C. Membrane Fission by Protein Crowding. Proc. Natl. Acad. Sci. U. S. A. 2017, 114, E3258-E3267.

(8) Fenz, S. F.; Sengupta, K. Giant Vesicles as Cell Models. Integr. Biol. 2012, 4, 982-995.

(9) Schwille, P. Jump-Starting Life? Fundamental Aspects of Synthetic Biology. J. Cell Biol. 2015, 210, 687-690. 
(10) Weiss, M.; Frohnmayer, J. P.; Benk, L. T.; Haller, B.; Janiesch, J.W.; Heitkamp, T.; Börsch, M.; Lira, R. B.; Dimova, R.; Lipowsky, R.; Bodenschatz, E.; Baret, J.-C.; Vidakovic-Koch, T.; Sundmacher, K.; Platzman, I.; Spatz, J. P. Sequential Bottom-up Assembly of Mechanically Stabilized Synthetic Cells by Microfluidics. Nat. Mater. 2017, 17, 89-96.

(11) Walde, P.; Cosentino, K.; Engel, H.; Stano, P. Giant Vesicles: Preparations and Applications. ChemBioChem 2010, 11, 848-865.

(12) van Swaay, D.; deMello, A. Microfluidic Methods for Forming Liposomes. Lab Chip 2013, 13, 752-767.

(13) Arriaga, L. R.; Datta, S. S.; Kim, S.-H.; Amstad, E.; Kodger, T. E.; Monroy, F.; Weitz, D. A. Ultrathin Shell Double Emulsion Templated Giant Unilamellar Lipid Vesicles with Controlled Microdomain Formation. Small 2014, 10, 950-956.

(14) Petit, J.; Polenz, I.; Baret, J.-C.; Herminghaus, S.; Baeumchen, O. Vesicles-on-a-chip: A Universal Microfluidic Platform for the Assembly of Liposomes and Polymersomes. Eur. Phys. J. E: Soft Matter Biol. Phys. 2016, 39, 16059.

(15) Blosser, M. C.; Horst, B. G.; Keller, S. L. cDICE Method Produces Giant Lipid Vesicles under Physiological Conditions of Charged Lipids and Ionic Solutions. Soft Matter 2016, 12, 7364-7371.

(16) Deshpande, S.; Birnie, A.; Dekker, C. On-Chip Density-Based Purification of Liposomes. Biomicrofluidics 2017, 11, 034106.

(17) Alam Shibly, S. U.; Ghatak, C.; Sayem Karal, M. A.; Moniruzzaman, M.; Yamazaki, M. Experimental Estimation of Membrane Tension Induced by Osmotic Pressure. Biophys. J. 2016, 111, 2190-2201.

(18) Jackman, J. A.; Kim, M. C.; Zhdanov, V. P.; Cho, N.-J. Relationship between Vesicle Size and Steric Hindrance Influences Vesicle Rupture on Solid Supports. Phys. Chem. Chem. Phys. 2016, 18, 3065-3072.

(19) Olbrich, K.; Rawicz, W.; Needham, D.; Evans, E. Water Permeability and Mechanical Strength of Polyunsaturated Lipid Bilayers. Biophys. J. 2000, 79, 321-327.

(20) Gauthier, N. C.; Masters, T. A.; Sheetz, M. P. Mechanical Feedback between Membrane Tension and Dynamics. Trends Cell Biol. 2012, 22, 527-535.

(21) Diz-Muñoz, A.; Fletcher, D. A.; Weiner, O. D. Use the Force: Membrane Tension as an Organizer of Cell Shape and Motility. Trends Cell Biol. 2013, 23, 47-53.

(22) Lipowsky, R. Spontaneous Tubulation of Membranes and Vesicles Reveals Membrane Tension Generated by Spontaneous Curvature. Faraday Discuss. 2013, 161, 305-331.

(23) Li, Y.; Lipowsky, R.; Dimova, R. Membrane Nanotubes Induced by Aqueous Phase Separation and Stabilized by Spontaneous Curvature. Proc. Natl. Acad. Sci. U. S. A. 2011, 108, 4731-4736.

(24) Aureli, M.; Mauri, L.; Ciampa, M. G.; Prinetti, A.; Toffano, G.; Secchieri, C.; Sonnino, S. GM1 Ganglioside: Past Studies and Future Potential. Mol. Neurobiol. 2016, 53, 1824-1842.

(25) Schengrund, C.-L. Gangliosides: Glycosphingolipids Essential for Normal Neural Development and Function. Trends Biochem. Sci. 2015, 40, 397-406.

(26) Ewers, H.; Römer, W.; Smith, A. E.; Bacia, K.; Dmitrieff, S.; Chai, W.; Mancini, R.; Kartenbeck, J.; Chambon, V.; Berland, L.; Oppenheim, A.; Schwarzmann, G.; Feizi, T.; Schwille, P.; Sens, P.; Helenius, A.; Johannes, L. GM1 Structure Determines SV40-Induced MembraneInvagination and Infection. Nat. Cell Biol. 2010, 12, 11-18.

(27) Bahrami, A. H.; Hummer, G. Formation and Stability of Lipid Membrane Nanotubes. ACS Nano 2017, 11, 9558-9565.

(28) Angelova, M.; Dimitrov, D. Liposome Electroformation. Faraday Discuss. Chem. Soc. 1986, 81, 303-311.

(29) Fricke, N.; Dimova, R. GM1 Softens POPC Membranes and Induces the Formation of Micron-Sized Domains. Biophys. J. 2016, 111, 1935-1945.

(30) Helfrich, W. Elastic Properties of Lipid Bilayers: Theory and Possible Experiments. Z. Naturforsch., C: J. Biosci. 1973, 28, 693-703.

(31) Seifert, U.; Berndl, K.; Lipowsky, R. Shape Transformations of Vesicles: Phase Diagram for Spontaneous Curvature and Bilayer
Coupling Model. Phys. Rev. A: At., Mol., Opt. Phys. 1991, 44, 11821202.

(32) Rand, R. P.; Burton, A. C. Mechanical Properties of the Red Cell Membrane I. Membrane Stiffness and Intracellular Pressure. Biophys. J. 1964, 4, 115-135.

(33) Hochmuth, R. M. Micropipette Aspiration of Living Cells. J. Biomech. 2000, 33, 15-22.

(34) Samudrala, N.; Nam, J.; Sarfati, R.; Style, R. W.; Dufresne, E. R. Mechanical Stability of Particle-Stabilized Droplets under Micropipette Aspiration. Phys. Rev. E: Stat. Phys., Plasmas, Fluids, Relat. Interdiscip. Top. 2017, 95, 012805 .

(35) Evans, E.; Yeung, A. Apparent Viscosity and Cortical Tension of Blood Granulocytes Determined by Micropipet Aspiration. Biophys. J. 1989, 56, 151-160.

(36) Tinevez, J.-Y.; Schulze, U.; Salbreux, G.; Roensch, J.; Joanny, J.F.; Paluch, E. Role of Cortical Tension in Bleb Growth. Proc. Natl. Acad. Sci. U. S. A. 2009, 106, 18581-18586.

(37) Agudo-Canalejo, J.; Lipowsky, R. Uniform and Janus-Like Nanoparticles in Contact with Vesicles: Energy Landscapes and Curvature-Induced Forces. Soft Matter 2017, 13, 2155-2173.

(38) Ringsdorf, H.; Schlarb, B.; Venzmer, J. Molecular Architecture and Function of Polymeric Oriented Systems: Models for the Study of Organization, Surface Recognition, and Dynamics of Biomembranes. Angew. Chem., Int. Ed. Engl. 1988, 27, 113-158.

(39) Needham, D.; Nunn, R. S. Elastic Deformation and Failure of Lipid Bilayer Membranes Containing Cholesterol. Biophys. J. 1990, 58, 997-1009.

(40) Discher, B. M.; Won, Y.-Y.; Ege, D. S.; Lee, J. C.-M.; Bates, F. S.; Discher, D. E.; Hammer, D. A. Polymersomes: Tough Vesicles Made from Diblock Copolymers. Science 1999, 284, 1143-1146.

(41) Jaskiewicz, K.; Larsen, A.; Schaeffel, D.; Koynov, K.; Lieberwirth, I.; Fytas, G.; Landfester, K.; Kroeger, A. Incorporation of Nanoparticles into Polymersomes: Size and Concentration Effects. ACS Nano 2012, 6, 7254-62.

(42) Dimova, R.; Seifert, U.; Pouligny, B.; Förster, S.; Döbereiner, H.G. Hyperviscous Diblock Copolymer Vesicles. Eur. Phys. J. E: Soft Matter Biol. Phys. 2002, 7, 241-250.

(43) Garten, M.; Mosgaard, L. D.; Bornschlögl, T.; Dieudonné, S.; Bassereau, P.; Toombes, G. E. S. Whole-GUV Patch-Clamping. Proc. Natl. Acad. Sci. U. S. A. 2017, 114, 328-333.

(44) Shchelokovskyy, P.; Tristram-Nagle, S.; Dimova, R. Effect of the HIV-1 Fusion Peptide on the Mechanical Properties and Leaflet Coupling of Lipid Bilayers. New J. Phys. 2011, 13, 025004. 